Relations industrielles

Industrial Relations

\title{
Répertoire de décisions en application du Code du travail, tome II, Mesures disciplinaires, par J.G. Descôteaux, Ottawa, Éditions de l’Université d'Ottawa, 1974, 268 p.
}

\section{Rodrigue Blouin}

Volume 30, numéro 4, 1975

URI : https://id.erudit.org/iderudit/028669ar

DOI : https://doi.org/10.7202/028669ar

Aller au sommaire du numéro

Éditeur(s)

Département des relations industrielles de l'Université Laval

ISSN

0034-379X (imprimé)

1703-8138 (numérique)

Découvrir la revue

Citer ce compte rendu

Blouin, R. (1975). Compte rendu de [Répertoire de décisions en application du Code du travail, tome II, Mesures disciplinaires, par J.G. Descôteaux, Ottawa, Éditions de l'Université d'Ottawa, 1974, 268 p.] Relations industrielles / Industrial Relations, 30(4), 800-801. https://doi.org/10.7202/028669ar

Tous droits réservés @ C Département des relations industrielles de l'Université Laval, 1975
Ce document est protégé par la loi sur le droit d'auteur. L'utilisation des services d’Érudit (y compris la reproduction) est assujettie à sa politique d'utilisation que vous pouvez consulter en ligne.

https://apropos.erudit.org/fr/usagers/politique-dutilisation/ 
souci constant de demeurer en contact avec le lecteur et de mettre celui-ci en situation d'action par rapport à l'organisation dont il peut être membre: «Un système de gestion fondé sur cette conception (l'approche-système) favorise une plus grande capacité d'agir, c'està-dire, plus de pouvoir pour les humains...» (p. 48).

La seconde partie, «Le système de gestion par objectifs, » est vraiment la partie-clef de cet ouvrage. Cette partie est essentiellement opérationnelle.

Dans un premier chapitre, l'auteur traite de la mission d'une organisation, des objectifs et des rôles reliés aux objectifs. Par la suite, il explique dans des chapitres distincts les différentes étapes à suivre pour analyser des situations organisationnelles, déterminer des objectifs, établir un plan d'action et le contrôler. Deux autres chapitres complètent cette partie. L'un qui présente «Un processus pour intégrer les éléments de la gestion », l'autre traitant de "L'implantation d'un système de gestion par objectifs».

Il existe beaucoup d'écrits sur le sujet de la gestion par objectifs ( MBO »). Peu se révèlent aussi près des réalités de l'organisation et des préoccupations quotidiennes des gestionnaires. Lefebvre explique étape par étape, illustre à l'occasion par des exemples simples et concrets, présente des grilles d'analyse, toujours avec un style direct qui conduit à l'action.

La troisième partie, «Sur l'art de la gestion » fournit un chapitre intéressant sur le travail d'équipe. Aujourd'hui, beaucoup d'organisations et de gestionnaires ont la hantise du travail d'équipe et des comités. La lecture de ce chapitre permet de désamorcer ce sujet, de le situer dans un contexte approprié et d'apprendre quand et comment utiliser le travail d'équipe.

Somme toute un excellent ouvrage, écrit et publié au Québec par un praticien du développement organisationnel; à notre connaissance c'est une première qu'il convient de souligner.

\section{Jean-Pierre BEAULIEU}

Alcan, Arvida
Répertoire de décisions en application du Code du travail, tome II, Mesures disciplinaires, par J. G. Descôteaux, Ottawa, Editions de l'Université d'Ottawa, 1974, $268 \mathrm{p}$.

Ce deuxième et dernier tome du Répertoire du professeur Descôteaux comblera un besoin certain dans l'ellaboration de synthèses d'ordre pédagogique et dans la recherche de solutions pratiques portant sur les problèmes soulevés par le congédiement pour activités syndicales (C. du t., art. 14 et ss.). S'il est en effet assez facile de connaître les décisions des commissaires-enquêteurs et jugements du tribunal du travail publiés depuis 1969 , il en va autrement pour la période antérieure. Le premier mérite de l'ouvrage de Me Descôteaux est d'avoir rassemblé et résumé les décisions rendues entre janvier 1962 et 1969 par les organismes alors compétents. L'importance de plusieurs de ces décisions peut être contestée même de nos jours, ne fusse que d'un point de vue comparatif. Le second mérite, moins perceptible, consiste à avoir regroupé pour fin de classification sous des thèmes précis les décisions et jugements rendus de 1969 à fin 1973. Le professeur Descôteaux comble ainsi un vide manifeste. La multiplicité des décisions rendues depuis janvier 1969 rendait en effet laborieuse l'identification des espèces jurisprudentielles selon les divers sujets particuliers que soulèvent l'application et l'interprétation des dispositions du Code sur le congédiement pour activités syndicales.

Les divers cas jurisprudentiels répertoriés dans l'ouvrage ont été regroupés en neuf grandes catégories générales codifiées comme suit: 100- Généralités; 200- Compétence des organismes; 300Mesures disciplinaires pour activités syndicales: congédiement, suspension, non-rappel, déplacement, mise à pied; 400- Réintégration et indemnité; 500 Fardeau de la preuve; 600- Revision de la décision; 700- Délai de soumission de la plainte; 800- Infractions pénales; 900- Requête pour permission d'appeler. Ces catégories ont elles-mêmes été divisées en autant de sous-catégories que le nécessitent la nature et la portée du prcblème. A titre d'exemple, la catégorie "Réintégration et indemnité 》 (no. cod. 400) donne lieu aux regroupements suivants: 400-01- Réintégration; 400-02-Indemnité; 400-03- Période servant à déterminer l'indemnité; 400-04- 
Critères de fixation du quantum; 400-05- Entente entre les parties; 400-06- Indemnité transmissible aux héritiers; 400-07-Cas de rappel au travail. Enfin, les sous-catégories sont elles-mêmes le plus souvent subdivisées en thèmes particuliers d'étude. A titre d'illustration, la sous-catégorie 100-01Notion de congédiement de la catégorie 100- Généralités présente les décisions en fonction des thèmes suivants: 100-11- Notion de suspension; 100-21Notion de lock-out; 100-31- Notion de mise à pied; 100-41- Notion de déplacement; 100-51- Notion de démission. Il suffira à l'utilisateur du Répertoire de quelques exercices pour découvrir les nombreux avantages de la méthode de codification utilisée par le professeur Descôteaux. Elle permet notamment, par un jeu de renvoi reposant sur le no. de codification, de signaler l'existence d'une même décision dans différents thèmes où ladite décision peut avoir une importance.

Les espèces jurisprudentielles répertoriées entre janvier 1962 et fin 1973 sont le résultat d'une analyse exhaustive. Le fait que Me Descôteaux ait procédé par l'étude des dossiers des différents organismes en est la garantie. Ces espèces sont présentées sous forme de sommaires. La démarche de rédaction est en principe la suivante: un bref rappel des faits; une mise en relief de l'objet du litige; la décision suivie de ses motifs; dégagement du principe résultant de la décision. Les utilisateurs du premier tome remarqueront que la démarche n'a pas subi de modification. On doit signaler que quelques-unes des espèces rapportées ne constituent plus de nos jours des guides, comme par exemple la fameuse affaire Bergeron (p. 21 du Répertoire); l'auteur a d'ailleurs invité le lecteur dans l'avant-propos à utiliser le volume en tenant compte des effets de l'écoulement du temps et, de plus, signalé généralement les décisions récentes infirmant celles de la période 1962-69 ou proposant une nouvelle orientation.

Les décisions et jugements rendus entre la période 1969 et fin 1973 ont été regroupés et classifiés selon le plan général de l'ouvrage. Ces décisions sont citées selon les besoins après les espèces résumées dans le cadre des sous-catégories ou thèmes. On ne présente cependant aucune idée du contenu de ces décisions. A la décharge de l'auteur, nous signalons que le rappel des cas jurisprudentiels récents n'est qu'un complément au Répertoire. Ce complément apparaît comme le premier essai visant à regrouper sous des thèmes précis les jugements rendus en application des articles 14 et ss. du C. du t.

Ce second tome du Répertoire nous apparaît comme une oeuvre colossale de synthèse des décisions des anciens organismes chargés de voir à l'application et à l'interprétation du Code du travail. Il s'agit d'une oeuvre importante qui ne peut échapper à l'attention de ceux qui s'intéressent aux problèmes soulevés par le congédiement pour activités syndicales. Nous espérons que cet ouvrage sera utilisé dans la recherche fondamentale et qu'il sera suivi de mises-à-jour périodiques.

\section{Rodrigue BLOUIN}

Université Laval

\section{Recommandations internationales sur les statistiques du travail par Bureau in- ternational du travail, Genève, 1975, $139 \mathrm{p}$.}

On sait que depuis sa création, l'Organisation internationale du travail a joué un rôle considérable pour normaliser les statistiques du travail. Le BIT a organisé une série de conférences internationales des statisticiens du travail; les résultats des travaux, d'abord présentés sous forme de résolutions pour approbation par le Conseil d'administration du BIT, ont par la suite été adoptés comme des recommandations.

Depuis la publication en 1959 de La normalisation internationale des statistiques du travail, trois Conférences internationales ont été tenues. Cet ouvrage vient présenter d'une façon systématique les principales recommandations actuellement en vigueur.

Il est divisé en dix chapitres: principales classifications économiques; maind'oeuvre, emploi, chômage et sous-emploi; salaire, durée du travail, coût de la main-d'oeuvre et revenu salarial; indices des prix à la consommation; enquêtes sur les conditions de la vie des familles; comparaisons internationales des salaires réels; sécurité sociale; lésicns professionnelles; conflits du travail; conventions collectives. Un annexe ccmprend la liste des Conférences internationales avec les questions qui $\mathrm{y}$ ont été examinées. 